\title{
Proposed terminology in fundamental astronomy based on IAU 2000 resolutions
}

\author{
Nicole Capitaine ${ }^{1}$, Alexandre H. Andrei ${ }^{2}$, Mark R. Calabretta ${ }^{3}$, \\ Véronique Dehant ${ }^{4}$, Toshio Fukushima ${ }^{5}$, Bernard R. Guinot ${ }^{1}$, \\ Catherine Y. Hohenkerk ${ }^{6}$, George H. Kaplan ${ }^{7}$, Sergei A. Klioner ${ }^{8}$, \\ Jean Kovalevsky ${ }^{9}$, Irina I. Kumkova ${ }^{10}$, Chopo Ma ${ }^{11}$, Dennis D. \\ McCarthy $^{7}$, Kenneth P. Seidelmann ${ }^{12}$ and Patrick T. Wallace ${ }^{13}$ \\ ${ }^{1}$ SYRTE, Observatoire de Paris, 61 Avenue de l'Observatoire, F-75014 Paris, France \\ email: n.capitaine@obspm.fr \\ ${ }^{2}$ Observatorio Nacional, R. Gal. Jose Cristino 77, São Cristóvão, Rio de Janeiro, \\ RJ 20921-400, Rio de Janeiro, Brazil \\ ${ }^{3}$ CSIRO / Australia Telescope National Facility, PO Box 76, Epping, NSW 1710, Australia \\ ${ }^{4}$ Royal Observatory Belgium, Avenue Circulaire 3, B-1180 Brussels, Belgium \\ ${ }^{5}$ National Astronomical Obesrevatory, 2-21-1 Osawa, Mitaka-shi, Tokyo 181-8588, Japan \\ ${ }^{6}$ HM Nautical Almanac Office, UK Hydrographic Office, \\ Admiralty Way, Taunton, Taunton TA1 2DN, UK \\ ${ }^{7}$ Astronomical Applications, US Naval Observervatory, \\ 3450 Massachusetts Ave NW, Washington DC 20392-5420, USA \\ ${ }^{8}$ Lohrmann Observatory, Technical University, D-01062 Dresden, Germany \\ ${ }^{9}$ ARTEMIS, Observatoire de la Côte d'Azur, Avenue N. Copernic, F-06130 Grasse, France \\ ${ }^{10}$ Sobolev Astronomical Institute, St Petersburg State University, \\ Dvortsovaya emb 18, RU-191186 St Petersburg, Russia \\ ${ }^{11}$ NASA Goddard Space Flight Center, Code 698, Greenbelt, MD 20771, USA \\ ${ }^{12}$ Virginia University, 129 Fontana Ct, Charlottesville, VA 22911-3531, USA \\ ${ }^{13}$ Department of Space Science \& Technology, STFC, Clilton, Didcot OX11 0QX, UK
}

\begin{abstract}
We present the proposals of the IAU Division I Working Group on Nomenclature for Fundamental Astronomy (NFA) that was formed at the IAU XXV General Assembly in 2003.
\end{abstract}

Keywords. reference system, time, ephemerides, standards, Earth

The NFA WG has worked on selecting a consistent and well defined terminology for all the quantities based on the IAU 2000 Resolutions on reference systems in order that it will be understood, recognized and adopted by the astronomical community.

We first recall the main nomenclature issues associated with the implementation of the IAU 2000 resolutions and especially those related to new concepts. We then report on the final NFA WG recommendations on terminology choices and guidelines that have been supported by explanatory documents. We finally discuss the resolution proposals to the IAU XXVI General Assembly, 2006, that have been prepared by the WG.

The IAU 2000 Resolutions recommended the use of an improved precession-nutation model, the use of a new definition of Universal Time and the adoption of a new origin on the equator as a replacement of the equinox. The NFA Recommendations related to these issues consist for example of specifying the terminology associated with the new paradigm, defining the celestial and terrestrial 'intermediate systems', keeping the 
classical terminology for 'true equator and equinox' and giving the name 'equation of the origins' to the distance between the Celestial intermediate origin (CIO) and the equinox along the intermediate equator. The NFA WG has also proposed a re-definition of Barycentric Dynamical Time (TDB) through a linear transformation of Barycentric Coordinate Time (TCB). These recommendations have resulted from a detailed discussion within the WG on issues described in a number of Newsletters and documents issued by the WG and posted on the NFA web site at ¡http://syrte.obspm.fr/iauWGnfa/i.

A special page of the NFA web site makes available documents with educational purposes relevant to the NFA issue.

The NFA explanatory document supplies information on the terminology and guidelines recommended by the WG. The NFA Glossary provides a set of detailed definitions that best explain all the terms required for implementing the IAU 2000 resolutions. The other sections provide complementary and supporting material to facilitate the understanding and implementation of the IAU resolutions, as well as illustrating the Glossary.

The NFA WG has submitted two resolution proposals to the IAU 2006 General Assembly; one is a 'Supplement to the IAU 2000 Resolutions on reference systems' for harmonizing the name of the pole and origin to 'intermediate' and fixing the default orientation of the BCRS and GCRS; the other one is a re-definition of TDB. 